\title{
(2) OPEN ACCESS \\ Implementation of a care bundle improves the management of patients with non-alcoholic fatty liver disease
}

For numbered affiliations see end of article.

\section{Correspondence to}

Dr Laura Jane Neilson, Department of Gastroenterology, South Tyneside and Sunderland NHS Foundation Trust, South Shields, NE34 OPL, UK; I. neilson@nhs.net

Received 4 April 2020 Revised 16 September 2020 Accepted 8 October 2020 Published Online First 4 January 2021

\section{Check for updates}

(C) Author(s) (or their employer(s)) 2021. Re-use permitted under CC BY-NC. No commercial reuse. See rights and permissions. Published by BMJ.

\section{To cite: Neilson LJ,}

Macdougall L, Lee PS, et al. Frontline Gastroenterology 2021;12:578-585.

\section{ABSTRACT}

Background Non-alcoholic fatty liver disease (NAFLD) is common and is associated with liverrelated and cardiovascular-related morbidity. Our aims were: (1) to review the current management of patients with NAFLD attending hospital clinics in North East England (NEE) and assess the variability in care; (2) develop a NAFLD 'care bundle' to standardise care; (3) to assess the impact of implementation of the NAFLD care bundle.

Methods A retrospective review was conducted to determine baseline management of patients with NAFLD attending seven hospitals in NEE. A care bundle for the management of NAFLD was developed including important recommendations from international guidelines. Impact of implementation of the bundle was evaluated prospectively in a single centre. Results Baseline management was assessed in 147 patients attending gastroenterology, hepatology and a specialist NAFLD clinic. Overall, there was significant variability in the lifestyle advice given and management of metabolic risk factors, with patients attending an NAFLD clinic significantly more likely to achieve $>10 \%$ body weight loss and have metabolic risk factors addressed. Following introduction of the NAFLD bundle 50 patients were evaluated. Use of the bundle was associated with significantly better documentation and implementation of most aspects of patient management including management of metabolic risk factors, documented lifestyle advice and provision of NAFLD-specific patient advice booklets.

\section{Significance of this study}

What is already known about this subject

- Non-alcoholic fatty liver disease (NAFLD) is an important cause of chronic liver disease that is associated with significant morbidity and mortality.

- Although hepatic complications as a result of cirrhosis are important, attention to metabolic risk factors is also necessary to reduce complications such as cardiovascular events.

- Appropriate staging of NAFLD is necessary to risk assess and guide management.

What this study adds

- This study demonstrates significant variability in the management of outpatients with NAFLD in North East England.

- NAFLD-specific treatment and weight loss advice were infrequently documented prior to implementation of a care bundle.

- Documentation and overall management improved following implementation of a simple care bundle.

Conclusion The introduction of an outpatient 'care bundle' led to significant improvements in the assessment and management of patients with NAFLD in the NEE and could help improve and standardise care if used more widely.

\section{BACKGROUND}

Non-alcoholic fatty liver disease (NAFLD) is an increasingly prevalent manifestation of obesity and the metabolic syndrome, 


\section{Significance of this study}

\section{How might it impact on clinical practice in the foreseeable future}

- Introduction of a care bundle into routine outpatient clinics may help improve and standardise the management of NAFLD.

- By ensuring all patients are staged appropriately this means NAFLD specific care can be targeted appropriately.

- Completion of the bundle may ensure that all metabolic risk factors are addressed.

and is now the most common cause of chronic liver disease in the UK and other developed countries. ${ }^{12}$ It is defined as fatty change in the liver affecting more than $5 \%$ of hepatocytes, in the absence of excessive alcohol consumption or steatogenic drugs. ${ }^{3}$ NAFLD ranges in severity from steatosis to non-alcoholic steatohepatitis (NASH) that can progress to cirrhosis and its complications, including hepatocellular carcinoma, liver failure and variceal haemorrhage. ${ }^{4-6}$ The severity of liver fibrosis in patients with NAFLD is a strong predictor of liver-related and all-cause mortality. ${ }^{78}$ Fibrosis staging is, therefore, critical to determine those who most need treatment or screening for liver-related complications. ${ }^{9}$

NAFLD is strongly associated with the metabolic syndrome,${ }^{10}$ defined as central obesity and at least two of: type 2 diabetes/raised fasting plasma glucose, hypertension, raised triglycerides and reduced high-density lipoprotein (HDL) cholesterol. ${ }^{11}$ The most frequent cause of death in patients with NAFLD is cardiovascular disease. Current management of NAFLD is focused on lifestyle modifications aimed at weight loss and increasing physical activity. ${ }^{12-14}$ Modest reductions in weight $(>5 \%)$ through lifestyle change have been shown to reduce steatosis and hepatic inflammation. ${ }^{15}{ }^{16}$ More marked weight loss (>10\%) can lead to liver fibrosis regression if sustained. ${ }^{15}$ Weight loss also improves metabolic health, quality of life and reduces all-cause mortality. ${ }^{17} 18$ Therefore, guidelines recommend targeting weight loss and increased physical activity as the primary treatment of NAFLD. ${ }^{12-14}$ It is also critical to appropriately manage associated metabolic features, such as diabetes, dyslipidaemia and hypertension, in order to reduce cardiovascular risk. ${ }^{12}$ Despite this, there are currently no national public health policies specifically for NAFLD and a previous UK audit demonstrated variation in approaches to the holistic management of patients with NAFLD and highlighted the limited access to lifestyle interventions. ${ }^{19} 20$ One recent study from Oxford demonstrated that a multidisciplinary metabolic hepatology clinic significantly improved the management of patients with NAFLD, leading to improved liver health and reduced cardiovascular risk. ${ }^{21}$ However, given that NAFLD is so prevalent, affecting $20 \%-25 \%$ of the UK population, it is unrealistic for all patients to have access to these specialist services. A simple approach to improve and standardise the general management of patients with NAFLD would therefore be advantageous. Care bundles have proven effective in a number of conditions, including decompensated liver cirrhosis where use of a bundle substantially improved care and reduced hospital stay. ${ }^{22} 23$

The aim of the current study was threefold. First, to assess the current management of patients with NAFLD attending outpatient clinics in hospitals in North East England (NEE). Second, to develop a simple care bundle for the management of patients with NAFLD to standardise care; and third, to assess the impact of implementation of the care bundle on the management of patients with NAFLD.

\section{METHODS}

This quality improvement project was delivered by the Gastroenterology Research and Audit by Northern Trainees (GRANT) network, a regional trainee research collaborative, and supported by the North East and North Cumbria Hepatology Network (NENCHN). The project comprised three phases: (1) baseline review of care of patients with NAFLD attending hospital clinics in NEE, (2) development of the NAFLD care bundle to standardise care and (3) implementation and audit of the impact of the care bundle on patient management. The project was registered and approved by the Newcastle upon Tyne Hospitals NHS Foundation Trust (NUTH) clinical governance department.

\section{Baseline regional review of NAFLD management}

This was a retrospective review of the management of NAFLD at seven hospitals in NEE. All hospitals in the region were invited to take part via the GRANT and NENCHN networks. Each site was asked to review the case notes of 20 patients with NAFLD attending for outpatient follow-up between January 2017 and June 2018. In NUTH, patients with NAFLD are seen in either a specialist NAFLD clinic or general hepatology clinics so 20 cases were collected from each clinic type. A data collection document was used to collect standardised data at all sites, which was transposed onto an anonymised spreadsheet and returned for central analysis. Data collection included: primary care management-investigations, basis of the NAFLD diagnosis, staging; secondary care management-documentation of alcohol consumption and metabolic risk factors, NAFLD staging (using Fibrosis-4 index (FIB-4), NAFLD fibrosis score, transient elastography or liver biopsy), documented lifestyle advice, metabolic risk factor management (diabetes, hypertension and dyslipidaemia) and NAFLD management. Performance was compared across clinics and statistical significance was assessed using Pearson's $\chi^{2}$ test or Fisher's exact test. Weight change between the patient's first clinic visit 


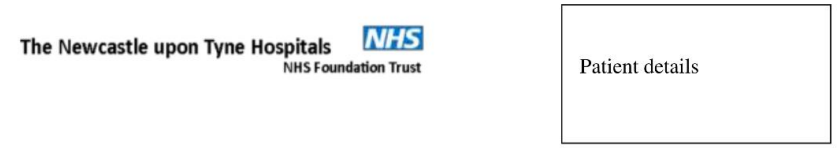

Care bundle: Management of patients with non-alcoholic fatty liver disease

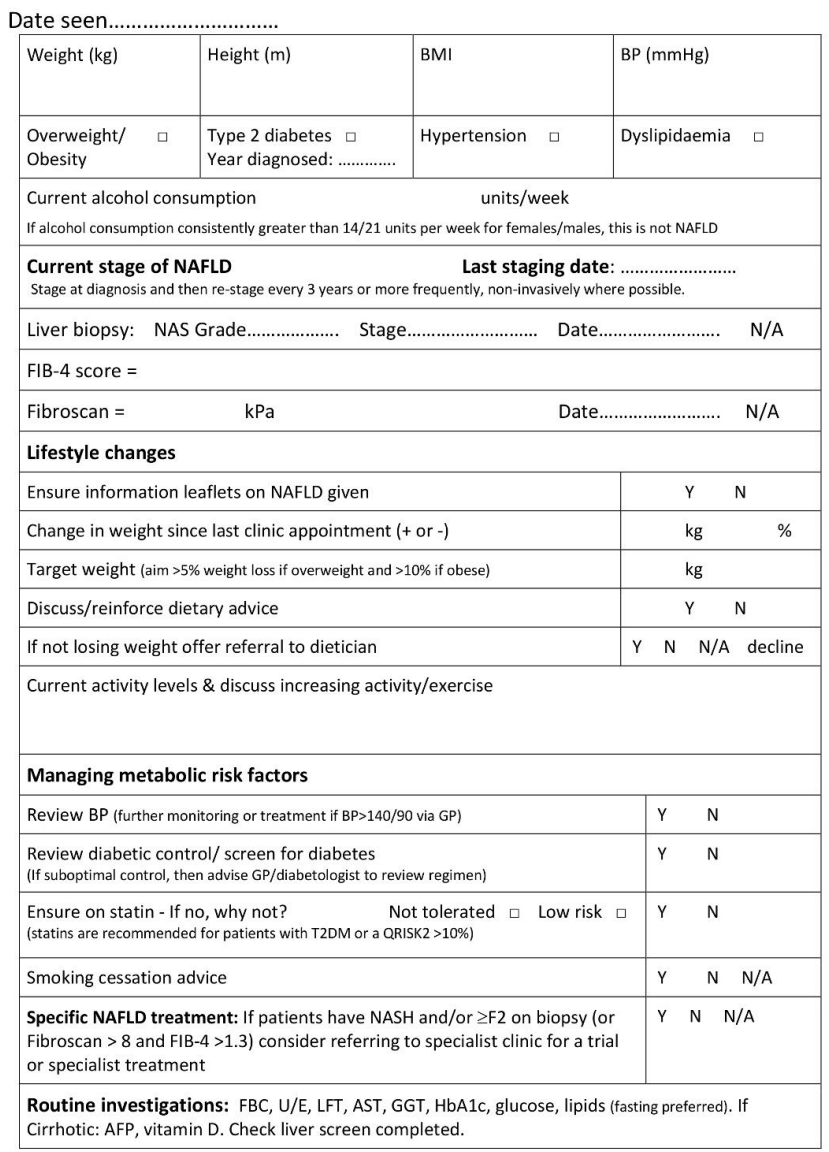

The Newcastle upon Tyne Hospitals WHS

Consultation:

Figure 1 Care bundle for management of patients with non-alcoholic fatty liver disease

and their latest visit was compared using Fisher's exact test.

\section{Development of the care bundle}

The care bundle was developed incorporating key recommendations from the European Association for the Study of the Liver (EASL) and National Institute for Health and Care Excellence (NICE) guidelines. ${ }^{12} 14$ The bundle (figure 1) provides a short structured 'check list' to facilitate the recording of anthropometry, metabolic risk factors, liver fibrosis stage, lifestyle advice and weight reduction targets, metabolic risk factor management and specific NAFLD treatment. It includes advice on routine investigations and an NAFLD management algorithm. The bundle was primarily aimed at NAFLD follow-up patients rather than those undergoing diagnostic work-up. The bundle was initially developed by two gastroenterology trainees (LJN and LMa) and one consultant hepatologist $(\mathrm{SMc})$ and then revised in response to critical review by a panel of experts including hepatologists, gastroenterologists, specialist dietician and exercise physiotherapist. Several cycles of review and revision were undertaken before the bundle was finalised.

\section{Review of outcomes following implementation of the NAFLD care bundle}

The care bundle was introduced at NUTH in November 2018. Paper copies of the bundle were made available in the outpatient department and the bundle was publicised at departmental meetings. All hepatologists and trainees were encouraged to use it when reviewing patients with NAFLD. A prospective audit was undertaken assessing consecutive follow-up patients with NAFLD attending general hepatology clinics involving three consultant hepatologists and four gastroenterology trainees between 14 January 2019 and 2 April 2019. Patients attending specialist NAFLD clinics were excluded as the bundle aimed to standardise care in a more general setting, where most variability was indicated by the baseline audit. Bundle completion was documented and data were collected on whether the key management aspects were addressed during the 
consultation. Comparisons between groups were made using Fisher's exact test and Mann-Whitney U tests.

\section{RESULTS}

Review of the management of patients with NAFLD in hospital clinics in NEE

A total of 147 patients (47\% male; median age 62; range 26-85 years) from 7 hospitals in the region contributed to this phase of the project. The median duration of follow-up between the first review and latest review was 23 months (range 2-221, SD 39.9). Overall, 68 patients (46\%) were identified from gastroenterology clinics, 59 (40\%) from hepatology clinics and 20 (14\%) from the Newcastle NAFLD clinic. The original referral was from primary care for $113(77 \%)$ patients and secondary care for 34 (23\%) patients. Most patients (74\%) were referred with abnormal liver blood tests. Other reasons for referral were evidence of steatosis on imaging $(15 \%)$, evidence of cirrhosis on imaging, abdominal pain and fatty liver identified at the time of cholecystectomy. In NEE, there has been a clearly defined referral pathway for patients with abnormal liver blood tests since $2014 .{ }^{24}$ This encompasses a blood liver screen (viral hepatitis, autoantibodies, coeliac screen, ferritin, random/fasting blood glucose or haemoglobin A1c (HbA1c and lipid screen) and non-invasive fibrosis assessment with FIB-4 score or NAFLD Fibrosis score prior to referral. Despite this, only $18 \%$ of patients had undergone a full blood liver screen in primary care, $40 \%$ had had a partial liver screen and only $16 \%$ of patients had non-invasive staging in primary care prior to referral.

At review in secondary care, an alcohol history, blood liver screen and liver ultrasound were undertaken in the majority of patients (see table 1). There was significant variability in the documentation of metabolic risk factors (obesity, blood pressure, lipids and diabetes) and staging of fibrosis within 3 years between the clinic types with higher rates of documentation of these areas in the NAFLD clinic (table 1). Overall, $53 \%$ of patients had diabetes, $65 \%$ were obese and 39\% were reported as having evidence of

Table 1 Comparison of the demographics and management of patients with non-alcoholic fatty liver disease (NAFLD) attending gastroenterology, hepatology and a specialist NAFLD clinic

\begin{tabular}{|c|c|c|c|c|c|}
\hline \multirow[b]{2}{*}{ Domain } & \multicolumn{3}{|l|}{ Clinic } & \multirow[b]{2}{*}{ Total (\%) } & \multirow[b]{2}{*}{$p$ value } \\
\hline & $\begin{array}{l}\text { Gastroenterology } \\
(\%) n=68\end{array}$ & $\begin{array}{l}\text { Hepatology } \\
\text { (\%) } n=59\end{array}$ & $\begin{array}{l}\text { NAFLD clinic }(\%) \\
\mathrm{n}=20\end{array}$ & & \\
\hline \multicolumn{6}{|l|}{ Patient characteristics } \\
\hline Sex (\% male) & $30(44 \%)$ & $32(54 \%)$ & $7(35 \%)$ & $69(47 \%)$ & - \\
\hline Diabetes \% & $34(50 \%)$ & $31(52 \%)$ & $13(65 \%)$ & $78(53 \%)$ & - \\
\hline Obesity (BMI >30 kg/m²) & $35(52 \%)$ & $43(73 \%)$ & $17(85 \%)$ & $95(65 \%)$ & - \\
\hline Cirrhosis \% & $27(40 \%)$ & $24(41 \%)$ & $6(30 \%)$ & $57(39 \%)$ & - \\
\hline Liver biopsy undertaken & $19(28 \%)$ & $26(44 \%)$ & $12(60 \%)$ & $57(39 \%)$ & - \\
\hline \multicolumn{6}{|l|}{ Interventions } \\
\hline Alcohol use documented* & $57(85 \%)$ & $54(92 \%)$ & $20(100 \%)$ & $131(90 \%)$ & 0.121 \\
\hline $\begin{array}{l}\text { Liver imaging (Ultrasound } \\
\text { scan)* }\end{array}$ & $64(96 \%)$ & $58(98 \%)$ & $20(100 \%)$ & $142(97 \%)$ & 0.519 \\
\hline Non-invasive staging* & $39(57 \%)$ & $40(68 \%)$ & $20(100 \%)$ & $99(67 \%)$ & 0.001 \\
\hline Staging within 3 years* & $29(45 \%)$ & $36(67 \%)$ & $17(85 \%)$ & $82(59 \%)$ & 0.003 \\
\hline Blood pressure checked* & $38(56 \%)$ & $36(61 \%)$ & $20(100 \%)$ & $94(65 \%)$ & 0.001 \\
\hline Lipids tested* & $43(64 \%)$ & $38(64 \%)$ & $20(100 \%)$ & $101(69 \%)$ & 0.005 \\
\hline Blood sugar addressed $t$ & $54(79 \%)$ & $40(68 \%)$ & $20(100 \%)$ & $114(78 \%)$ & 0.004 \\
\hline $\begin{array}{l}\text { BMI documented at review } \\
\text { appointment* }\end{array}$ & $47(70 \%)$ & $35(60 \%)$ & $4(20 \%) \ddagger$ & $86(59 \%)$ & $<0.001$ \\
\hline $\begin{array}{l}\text { Waist circumference } \\
\text { documented } t\end{array}$ & $0(0 \%)$ & $1(2 \%)$ & $20(100 \%)$ & $21(14 \%)$ & $<0.001$ \\
\hline $\begin{array}{l}\text { Weight loss advice } \\
\text { documentedt }\end{array}$ & $46(68 \%)$ & $51(86 \%)$ & $19(95 \%)$ & $116(79 \%)$ & 0.006 \\
\hline $\begin{array}{l}\text { Documented advice to lose } \\
\geq 10 \% \text { body weight } \dagger\end{array}$ & $6(9 \%)$ & $4(7 \%)$ & $5(25 \%)$ & $15(10 \%)$ & 0.084 \\
\hline Statin prescribed* & $35(56 \%)$ & $31(61 \%)$ & $16(80 \%)$ & $82(61 \%)$ & 0.153 \\
\hline $\begin{array}{l}\text { Referred to structured lifestyle } \\
\text { programmet }\end{array}$ & $6(9 \%)$ & $4(7 \%)$ & $11(55 \%)$ & $21(15 \%)$ & $<0.001$ \\
\hline
\end{tabular}

*Pearson $\chi^{2}$ test was used.

tFisher's exact test was used.

¥Although body mass index (BMI) not formally documented, height and weight were documented for all patients. 
Table 2 Comparison of weight loss between the first and current clinic review between the specialist non-alcoholic fatty liver disease (NAFLD) and non-specialist clinics (general gastroenterology and hepatology clinics)

\begin{tabular}{llll}
\hline $\begin{array}{l}\text { Actual weight } \\
\text { loss }\end{array}$ & $\begin{array}{l}\text { Non-specialist } \\
\text { clinics }\end{array}$ & NAFLD & p value \\
\hline Any & $63(56 \%)$ & $15(75 \%)$ & 0.091 \\
$>5 \%$ & $24(27 \%)$ & $8(40 \%)$ & 0.071 \\
$>10 \%$ & $7(6 \%)$ & $5(25 \%)$ & $\mathbf{0 . 0 1 9}$ \\
\hline
\end{tabular}

Bold values are those which reached statistical significance.

cirrhosis. Documentation of body mass index was variable, including in the specialist NAFLD clinic.

Given that weight loss is the recommended treatment for NAFLD, this is an important measure of success of treatment. Weight loss advice and weight loss targets were generally inadequately documented, with varying recommendations. When compared with patients attending non-specialist clinics, those attending the specialist NAFLD clinic were significantly more likely to have lost $>10 \%$ of their body weight $(\mathrm{p}=0.019$. table 2), a degree of weight loss shown to be associated with improved liver fibrosis. ${ }^{15}$ It was not possible from this audit to identify specific weight loss or dietary strategies.

\section{Review of the impact of implementation of the care bundle on management of NAFLD}

Following implementation of the bundle, 50 consecutive patients with NAFLD attending four hepatology clinics were identified. The median age was 61 (range 22-82 years), $60 \%$ were male, $60 \%$ had the full metabolic syndrome and the majority were originally referred from primary care (66\%). All patients had fibrosis staging with FIB-4 score, transient elastography or liver biopsy (32\%) and 34\% had cirrhosis. The care bundle was used in $46 \%$ of clinic encounters. A comparison of the baseline demographics and documentation of key aspects of care is shown between those with and without a completed bundle in table 3 . In general, use of the bundle was associated with significantly better documentation and implementation of most aspects of patient management. In particular, the documentation of management of metabolic risk factors, documented lifestyle advice and provision of patient information booklets on NAFLD was better when the bundle was completed.

\section{DISCUSSION}

This regional review in NEE showed significant variability in the management of patients with NAFLD. Documentation of the active management of NAFLD, particularly lifestyle interventions and management of metabolic risk factors, was poor in general gastroenterology and hepatology clinics. Given the increasing prevalence of NAFLD and limited specialist NAFLD clinic capacity, there is a real need to standardise and improve care of patients with NAFLD to ensure all affected individuals are managed in accordance with National Institute for Health and Care Excellence (NICE) and European Association for the Study of the

Table 3 Review of outcomes following implementation of the NAFLD bundle

\begin{tabular}{|c|c|c|c|}
\hline & $\begin{array}{l}\text { NAFLD bundle completed } \\
\mathrm{n}=23\end{array}$ & $\begin{array}{l}\text { NAFLD bundle not completed } \\
\mathrm{n}=27\end{array}$ & $\mathrm{p}$ value \\
\hline Consultant review (ie, not trainee) & $52 \%$ & $48 \%$ & \\
\hline Age (years)* & $60(22-82)$ & $63(30-81)$ & 0.43 \\
\hline Sex (\% male) & $57 \%$ & $63 \%$ & 0.77 \\
\hline Body Mass Index* & $33.8(25.9-52)$ & $35(29-44)$ & 0.69 \\
\hline Stage 3 or 4 fibrosis & $61 \%$ & $44 \%$ & 0.27 \\
\hline \multicolumn{4}{|l|}{ Metabolic risk factors } \\
\hline Obesity & $96 \%$ & $84 \%$ & 0.31 \\
\hline Type 2 diabetes mellitus & $61 \%$ & $56 \%$ & 1.0 \\
\hline Hypertension & $61 \%$ & $56 \%$ & 1.0 \\
\hline Dyslipidaemia & $83 \%$ & $85 \%$ & 1.0 \\
\hline \multicolumn{4}{|l|}{ Interventions } \\
\hline Target weight documented & $78 \%$ & $22 \%$ & $<0.001$ \\
\hline Blood pressure reviewed & $78 \%$ & $22 \%$ & $<0.001$ \\
\hline Diabetes reviewed & $100 \%$ & $44 \%$ & $<0.001$ \\
\hline Dyslipidaemia reviewed & $100 \%$ & $78 \%$ & 0.016 \\
\hline Alcohol intake documented & $87 \%$ & $41 \%$ & 0.001 \\
\hline Patient provided with NAFLD information booklet & $96 \%$ & $11 \%$ & $<0.001$ \\
\hline Dietary advice documented & $100 \%$ & $59 \%$ & 0.001 \\
\hline Exercise advice documented & $96 \%$ & $56 \%$ & 0.001 \\
\hline
\end{tabular}

*Mann-Whitney U test used.

BMI, body mass index. 
Liver (EASL) recommendations. ${ }^{12} 1419$ In response to these findings, we have developed a 'care bundle' to help improve the care of patients with NAFLD. The bundle provides a standardised approach to record key information such as anthropometry, stage of liver disease and metabolic risk factors. Moreover, the care bundle provides a treatment algorithm and a checklist to ensure lifestyle advice and treatment goals are more clearly documented, and metabolic risk factors are actively managed. Our study of the care bundle implementation in general hepatology clinics demonstrated that in individuals where the care bundle was used, key aspects of management were significantly more likely to be addressed and documented. This suggests that more widespread use of the bundle could improve outcomes for patients with NAFLD and a larger assessment of the impact of the bundle on longterm outcomes is warranted.

The regional audit demonstrated relatively low levels of active management of NAFLD in non-specialist clinics and few patients achieved significant weight loss. This may suggest that clinicians focus on making a diagnosis of the cause of raised liver enzymes rather than actively managing NAFLD. ${ }^{25}$ More active management of patients with NAFLD is needed to reduce the risk of patients progressing to advanced liver fibrosis/ cirrhosis, especially among those with type 2 diabetes who are at the highest risk of fibrosis progression. ${ }^{5}$ Moreover, patients with NAFLD are at high risk of cardiovascular disease with approximately $40 \%$ dying a cardiovascular death, so actively addressing cardiovascular risk in clinic is vital to improve long-term patient outcomes. ${ }^{67}$ While we were unable to identify specific weight loss strategies or dietary advice in this study, approaches such as the Mediterranean diet have been shown to reduce liver fat in the absence of weight loss. ${ }^{26}$ Our review shows that patients attending the specialist NAFLD clinic have active management of lifestyle change and metabolic risk and this translates into better objective outcomes with patients being significantly more likely to lose $>10 \%$ body weight, which is known to improve liver histology in patients with NASH. ${ }^{15}$ While this can be attributed in part to better access to structured lifestyle programmes, these clinics are more focused on active management of the condition rather than just making a diagnosis. The care bundle was designed to prompt clinicians to consider and identify metabolic risk factors, such as diabetes and hypertension, but does not recommend specific treatment. We hope that the care bundle will promote a 'culture change' in non-specialist clinics by giving a more structured and checklist approach to more active management of lifestyle change and modification of cardiovascular risk. Licensing of specific pharmacological treatments for NAFLD is anticipated in the near future and the bundle could be adapted to include these NAFLD specific treatments.
Another important finding from the regional review was that despite there being clearly defined regional guidance on the investigation of raised liver blood tests for primary care since 2014 , only $18 \%$ had a full blood liver screen and $16 \%$ had non-invasive staging with the FIB-4 score or NAFLD fibrosis score completed prior to clinic referral. While initial referral may have preceded these guidelines in some cases, guideline compliance is disappointingly low and suggests a need for further education in primary care. The care bundle could also be adapted for use in primary care to improve compliance with guidelines.

Overall, uptake of the bundle was suboptimal, being completed in only $46 \%$ of clinic consultations. We have not formally assessed the reasons for this but informal discussion with clinicians participating have indicated that reasons for non-use of the bundle included a perception of increased time required for bundle completion in already busy clinics. However, once clinicians became familiar with it, many recognised its benefits in providing structure and focus to the consultation to ensure important aspects were addressed. It is well known that implementation of new approaches can take time to embed in clinical practice. This was seen with the previous introduction of the decompensated cirrhosis care bundle. ${ }^{23}$

This review has some limitations. First, the regional review and implementation of the NAFLD bundle were conducted by medical case note review and as such included what was recorded in the notes, which may not have been reflective of actual discussions in the clinic appointment. However, we noted significant differences in objective outcomes, such as weight change, between the clinics and these were consistent with all the measured outcomes, suggesting that significant variation is real. Second, the NAFLD bundle implementation study was conducted at a single centre with a relatively small sample size that could have been influenced by the practice of specific clinicians. However, seven doctors (three consultants and four specialist trainees) were involved and equal proportions of patients in each group were seen by consultants and trainees. Third, long-term outcomes of the impact of the bundle have not yet been assessed. To confirm our findings and assess the long-term impact on outcomes, rollout of the NAFLD bundle is planned on a larger scale across the whole of NEE with prospective data collection.

In conclusion, we found significant variability in the management of patients with NAFLD attending hospitals in NEE, confirming a clear need to improve the holistic management of these patients. In response to this, we developed a care bundle to standardise care and promote the active management of NAFLD and its associated metabolic risk factors. Our data have demonstrated that use of the NAFLD bundle significantly improved the holistic care of patients with NAFLD. 


\section{Author affiliations}

${ }^{1}$ Liver Unit, Newcastle Upon Tyne Hospitals NHS Foundation Trust, Newcastle Upon Tyne, UK

${ }^{2}$ Department of Gastroenterology, South Tyneside and Sunderland NHS Foundation Trust, South Shields, UK

${ }^{3}$ Department of Gastroenterology, University Hospital of North Tees, Stocktonon-Tees, UK

${ }^{4}$ Department of Gastroenterology, University Hospital of North Durham, Durham, UK

${ }^{5}$ Department of Gastroenterology, Gateshead Health NHS Foundation Trust, Gateshead, UK

${ }^{6}$ Department of Gastroenterology, Northumbria Healthcare NHS Foundation Trust, North Shields, UK

${ }^{7}$ Department of Gastroenterology, Darlington Memorial Hospital, Darlington, UK ${ }^{8}$ Translational and Clinical Research Institute, Newcastle University, Newcastle upon Tyne, UK

\section{Twitter Dina Mansour@drdina_mansour}

Contributors SMc conceived the study and oversaw data collection, analysis and interpretation. SMc and LJN designed the study. SMc, LJN and LM designed the 'care bundle.' LJN collected data and coordinated regional data collection for the initial audit. LM, DB, SC, AE, MH, SG, SL, SO, NAP and WT collected regional data. PSSL and TH collected data for the subsequent audit. LH, KH, SM, DM, QA and JKD critically reviewed the 'care bundle.' All authors contributed to study design, critically reviewed manuscript drafts and approved the final article for submission. SMc is guarantor for the data.

Funding The authors have not declared a specific grant for this research from any funding agency in the public, commercial or not-for-profit sectors.

Competing interests $\mathrm{SMcP}$ has no conflicts of interest related to this work, but has acted as a speaker or advisory board/ consultancy for Abbvie, Allergan, Gilead, Intercept, Merk, Sequana. QA has received research grant funding from Abbvie, Allergan/Tobira, AstraZeneca, GlaxoSmithKline, Glympse Bio, Novartis Pharma AG, Pfizer Ltd., Vertex. He has active research collaborations with (including research collaborations supported through the EU IMI2 LITMUS Consortium*) Abbvie, Antaros Medical*, Allergan/Tobira*, AstraZeneca*, BMS*, Boehringer Ingelheim International GMBH*, Echosens*, Ellegaard Gottingen Minipigs AS*, Eli Lilly \& Company Ltd.*, Exalenz Bioscience Ltd.*, Genfit SA*, Glympse Bio, GlaxoSmithKline, HistoIndex", Intercept Pharma Europe Ltd.*, iXscient Ltd.*, Nordic Bioscience*, Novartis Pharma AG*, Novo Nordisk A/S*, One Way Liver Genomics SL*, Perspectum Diagnostics*, Pfizer Ltd.*, Resoundant*, Sanofi-Aventis Deutschland GMBH*, SomaLogic Inc.", Takeda Pharmaceuticals International SA*. He has provided consultancy to 89Bio, Abbott Laboratories, Acuitas Medical, Allergan/Tobira, Altimmune, AstraZeneca, Axcella, Blade, BMS, BNN Cardio, Celgene, Cirius, CymaBay, EcoR1, E3Bio, Eli Lilly \& Company Ltd., Galmed, Genentech, Genfit SA, Gilead, Grunthal, HistoIndex, Indalo, Imperial Innovations, Intercept Pharma Europe Ltd., Inventiva, IQVIA, Janssen, Madrigal, MedImmune, Metacrine, NewGene, NGMBio, North Sea Therapeutics, Novartis, Novo Nordisk A/S, Pfizer Ltd., Poxel, ProSciento, Raptor Pharma, Servier, Terns, Viking Therapeutics. He has been a speaker for Abbott Laboratories, Allergan/Tobira, BMS, Clinical Care Options, Falk, Fishawack, Genfit SA, Gilead, Integritas Communications, Kenes,

MedScape. He receives royalties from Elsevier Ltd (Davidson's Principles \& Practice of Medicine textbook). No other authors have COI to declare.

Patient consent for publication Not required.

Ethics approval This quality improvement project was registered and approved by the Newcastle upon Tyne Hospitals NHS Foundation Trust (NUTH) Clinical Governance Department (project number 8268).

Provenance and peer review Not commissioned; externally peer reviewed.

Data availability statement No data are available.
Open access This is an open access article distributed in accordance with the Creative Commons Attribution Non Commercial (CC BY-NC 4.0) license, which permits others to distribute, remix, adapt, build upon this work noncommercially, and license their derivative works on different terms, provided the original work is properly cited, appropriate credit is given, any changes made indicated, and the use is noncommercial. See: http://creativecommons.org/licenses/by-nc/4. $0 /$.

\section{ORCID iDs}

Laura Jane Neilson http://orcid.org/0000-0002-7185-0825

Phey Shen Lee http://orcid.org/0000-0002-6007-8227

Subashini Chandrapalan http://orcid.org/0000-0002-15823224

\section{REFERENCES}

1 Anstee QM, McPherson S, Day CP. How big a problem is nonalcoholic fatty liver disease? BMJ 2011;343:d3897.

2 Estes C, Anstee QM, Arias-Loste MT, et al. Modeling NAFLD disease burden in China, France, Germany, Italy, Japan, Spain, United Kingdom, and United States for the period 2016-2030. J Hepatol 2018;69:896-904.

3 Gallacher J, McPherson S. Practical diagnosis and staging of nonalcoholic fatty liver disease: a narrative review. Eur Med J 2018;3:108-18.

4 Wanless IR, Lentz JS. Fatty liver hepatitis (steatohepatitis) and obesity: an autopsy study with analysis of risk factors. Hepatology 1990;12:1106-10.

5 McPherson S, Hardy T, Henderson E, et al. Evidence of NAFLD progression from steatosis to fibrosing-steatohepatitis using paired biopsies: implications for prognosis and clinical management. J Hepatol 2015;62:1148-55.

6 Vilar-Gomez E, Calzadilla-Bertot L, Wai-Sun Wong V, et al. Fibrosis severity as a determinant of cause-specific mortality in patients with advanced nonalcoholic fatty liver disease: a Multi-National cohort study. Gastroenterology 2018;155:44357.

7 Hagström H, Nasr P, Ekstedt M, et al. Fibrosis stage but not NASH predicts mortality and time to development of severe liver disease in biopsy-proven NAFLD. J Hepatol 2017;67:1265-73.

8 Taylor RS, Taylor RJ, Bayliss S, et al. Association between fibrosis stage and outcomes of patients with nonalcoholic fatty liver disease: a systematic review and meta-analysis. Gastroenterology 2020;158:1611-25.

9 Dyson JK, Anstee QM, McPherson S. Republished: nonalcoholic fatty liver disease: a practical approach to treatment. Postgrad Med J 2015;91:92-101.

10 Anstee QM, Targher G, Day CP. Progression of NAFLD to diabetes mellitus, cardiovascular disease or cirrhosis. Nat Rev Gastroenterol Hepatol 2013;10:330-44.

11 Alberti KGMM, Eckel RH, Grundy SM, et al. Harmonizing the metabolic syndrome: a joint interim statement of the International diabetes Federation Task force on epidemiology and prevention; National heart, lung, and blood Institute; American heart association; world heart Federation; international atherosclerosis Society; and international association for the study of obesity. Circulation 2009; 120:1640-5.

12 European Association for the Study of the Liver (EASL), European Association for the Study of Diabetes (EASD), European Association for the Study of Obesity (EASO). EASLEASD-EASO clinical practice guidelines for the management of non-alcoholic fatty liver disease. J Hepatol 2016;64:1388-402.

13 Chalasani N, Younossi Z, Lavine JE, et al. The diagnosis and management of nonalcoholic fatty liver disease: practice guidance from the American association for the study of liver diseases. Hepatology 2018;67:328-57. 
14 National Institute for Health and Care Excellence. NonAlcoholic fatty liver disease (NAFLD): assessment and management (NICE guideline 49). retrieved from, 2016. Available: www.nice.org.uk/guidance/ng49

15 Vilar-Gomez E, Martinez-Perez Y, Calzadilla-Bertot L, et al. Weight loss through lifestyle modification significantly reduces features of nonalcoholic steatohepatitis. Gastroenterology 2015;149:367-78.

16 Harrison SA, Fecht W, Brunt EM, et al. Orlistat for overweight subjects with nonalcoholic steatohepatitis: a randomized, prospective trial. Hepatology 2009;49:80-6.

17 Look AHEAD Research Group, Gregg E, Jakicic J, et al. Association of the magnitude of weight loss and changes in physical fitness with long-term cardiovascular disease outcomes in overweight or obese people with type 2 diabetes: a post-hoc analysis of the look ahead randomised clinical trial. Lancet Diabetes Endocrinol 2016;4:913-21.

18 Lean ME, Leslie WS, Barnes AC, et al. Primary care-led weight management for remission of type 2 diabetes (direct): an openlabel, cluster-randomised trial. Lancet 2018;391:541-51.

19 Sheridan DA, Aithal G, Alazawi W, et al. Care standards for non-alcoholic fatty liver disease in the United Kingdom 2016: a cross-sectional survey. Frontline Gastroenterol 2017;8:252-9.

20 Lazarus JV, Ekstedt M, Marchesini G, et al. A cross-sectional study of the public health response to non-alcoholic fatty liver disease in Europe. J Hepatol 2020;72:14-24.
21 Moolla A, Motohashi K, Marjot T, et al. A multidisciplinary approach to the management of NAFLD is associated with improvement in markers of liver and cardio-metabolic health. Frontline Gastroenterol 2019;10:337-46.

22 McPherson S, Dyson J, Austin A, et al. Response to the NCEPOD report: development of a care bundle for patients admitted with decompensated cirrhosis-the first $24 \mathrm{~h}$. Frontline Gastroenterol 2016;7:16-23.

23 Dyson JK, Rajasekhar P, Wetten A, et al. Implementation of a "care bundle" improves the management of patients admitted to hospital with decompensated cirrhosis.[Erratum appears in Aliment Pharmacol Ther. 2017 Feb;45(4):579; PMID: 28074508]. Aliment Pharmacol Ther 2016;44:1030-8.

24 Hudson M, Mcpherson S. Liver Network Guidelines for the Management of Adults with Asymptomatic Liver Function Abnormalities On behalf of the North East \& North Cumbria Hepatology Network 2016.

25 Avery L, Exley C, McPherson S, et al. Lifestyle behavior change in patients with nonalcoholic fatty liver disease: a qualitative study of clinical practice. Clin Gastroenterol Hepatol 2017;15:1968-71.

26 Romero-Gómez M, Zelber-Sagi S, Trenell M. Treatment of NAFLD with diet, physical activity and exercise. J Hepatol 2017;67:829-46. 